\title{
A two-dimensional simulation of thermospheric vertical winds in the vicinity of an auroral arc
}

\author{
H. Shinagawa ${ }^{1}$ and S. Oyama ${ }^{2 *}$ \\ ${ }^{1}$ National Institute of Information and Communications Technology, Koganei, Tokyo 184-8795, Japan \\ ${ }^{2}$ Geophysical Institute, University of Alaska Fairbanks, Fairbanks, AK 99775-7320, USA
}

(Received January 16, 2006; Revised June 28, 2006; Accepted September 7, 2006; Online published September 29, 2006)

\begin{abstract}
The observations made by Fabry-Perot interferometers (FPIs), radars, and satellites have indicated that large vertical motion in the polar region is occasionally generated in the thermosphere associated with auroral activities. However, the behavior of the vertical wind is often very complicated, and the cause of the vertical wind has not been explained by auroral heating or by ion-neutral drag alone. It has been pointed out that a background horizontal flow is likely to significantly alter the dynamics of the neutral atmosphere near an auroral arc. Recent observations have also suggested that strong downward motion is generated in the vicinity of an auroral arc. To study the thermospheric dynamics near a local heating region embedded in a large-scale horizontal flow, a twodimensional numerical simulation of the thermospheric dynamics has been performed. It is found that interaction of local heating and strong horizontal flow could play an important role in generating vertical motion near an auroral arc. The simulation results indicate that for a horizontal wind speed larger than about $300 \mathrm{~m} / \mathrm{s}$, a steady wave-like structure of the neutral wind is formed within and downstream of the heated region. For a horizontal wind speed less than about $300 \mathrm{~m} / \mathrm{s}$, on the other hand, no significant vertical motion is generated outside the heated region. This process might account for at least some of the observed features of vertical motion within and outside an auroral arc.
\end{abstract}

Key words: Thermosphere, vertical wind, auroral heating, simulation.

\section{Introduction}

Effects of the auroral activity on thermospheric motions have been studied extensively using optical and radio techniques and theoretical calculations. Theoretical studies as well as observations indicate that global-scale convection in the thermosphere is driven by ion-neutral drag force through collisions between ion and neutral particles (Killeen et al., 1985; McCormac et al., 1985; Rees et al., 1986; Thayer et al., 1987). Effects of auroral heating on the thermospheric wind system have been studied using numerical simulation of the upper atmosphere (Richmond and Matsushita, 1975; Hajkowicz and Hunsucker, 1987; Maeda et al., 1989; Fujiwara et al., 1996). The global-scale models, however, do not always provide realistic structures of the polar thermosphere and ionosphere associated with small-scale structures of the auroral arc. In particular, thermospheric motions in the vicinity of small-scale auroral structures are quite sensitive not only to magnitude but also to spatial and temporal variations in the electric field, the plasma density, and the heating rate (Fuller-Rowell, 1984, 1985; Walterscheid et al., 1985; Sun et al., 1995).

Recent observations made by ground-based Fabry-Perot interferometers (FPIs) have shown latitudinal shear of the

\footnotetext{
* Now at Solar-Terrestrial Environmental Laboratory, Nagoya University, Chikusa-ku, Nagoya 464-8601, Japan.

Copyright (c) The Society of Geomagnetism and Earth, Planetary and Space Sciences (SGEPSS); The Seismological Society of Japan; The Volcanological Society of Japan; The Geodetic Society of Japan; The Japanese Society for Planetary Sciences; TERRAPUB
}

geomagnetic zonal wind in the premidnight time sector in association with both the equatorward and poleward boundaries of the discrete aurora (Conde et al., 2001). These zonal winds are considered to be driven by geomagnetically westward ion convection. Because the direction of the observed westward neutral winds is in opposition to the global-scale pressure gradient caused by solar heating, the momentum transfer from ions to neutrals through collisions prevails the pressure gradients in the conducting ionosphere caused by auroral particle precipitation. Model calculations showed that the ion-drag acceleration of neutral wind could lead to the development of significant horizontal wind shears (Lyons and Walterscheid, 1985; Parish et al., 2003).

In addition to horizontal winds, local vertical winds in the auroral region have been observed with FPIs and Dynamics Explorer (DE) spacecraft (Spencer et al., 1981; Rees et al., 1984; Sica et al., 1986; Eastes et al., 1992; Price et al., 1995; Conde and Dyson, 1995; Johnson et al., 1995; Smith and Hernandez, 1995; Smith, 2000; Ishii et al., 2001; Innis and Conde, 2002; Ishii, 2005).

A number of studies indicated that the vertical neutral motion could cause major changes in neutral composition as well as ion composition in the F-region and upper E-region ionosphere. Changes in the neutral composition affect the ionospheric recombination rate and the lifetime of ionospheric plasma. The heat balance in the neutral atmosphere is also influenced by the neutral composition. Theoretically, Sojka et al. (2001) investigated effects of the upward neutral flow on the ionosphere using a theoretical model, suggest- 
ing that significant ionospheric displacement could occur associated with the upward motion of the thermosphere.

The behavior of the vertical wind, especially the significant downward wind, has not been explained simply by Joule and particle heating processes, or vertical component of ion drag force, suggesting that complex mechanisms play an important role in the formation of vertical winds in the upper thermosphere. Burnside et al. (1981) suggested that the divergent component of strong variations in the horizontal wind field is connected to the vertical winds. However, specific cause of local variations in the horizontal wind has not been presented specifically. Smith (2000) suggested that a vertical wave-like motion could be generated in the downstream region of the heat source through a local heating within a strong horizontal flow. The observations by DE-2 imply such a mechanism of generation of the vertical wind (Innis and Conde, 2002). The time-dependent thermospheric response to the energy input over small horizontal scale during high auroral active periods has been simulated with sophisticated two- and threedimensional high-resolution models (Fuller-Rowell, 1984, 1985; Walterscheid et al., 1985; Walterscheid and Lyons, 1989, 1992; Sun et al., 1995). However, strong vertical motion, especially downward motion has not been reproduced by those models. Shinagawa et al. (2003) studied the dynamics near a moving auroral arc, using a two-dimensional thermospheric model. They found that wave-like signature tends to be formed in the downstream region of the moving heat source. However, their study showed only a few snapshots of the velocity near an arc, which might not be a typical or steady feature. Therefore, interaction between local dynamics driven by small-scale heat source and horizontal flow needs to be studied in more detail.

In this study, we examine whether or not and how significant vertical oscillation suggested by Smith (2000) and by Shinagawa et al. (2003) is generated as a result of interaction of local heating and background horizontal wind field. To solve the fluid equations for the neutral atmosphere, a relatively new numerical technique, Cubic-Interpolated Pseudo-particle (CIP) method, is appllied, and the basic procedure is briefly described in Appendix.

\section{Simulation Model}

In the thermospheric dynamics near an auroral arc, the vertical scale of disturbance becomes comparable to the horizontal scale of disturbance. The typical time scale of variations associated with auroral activities is usually comparable or even less than the Brunt-Väisälä period. In addition, the horizontal wind speed of the thermosphere reaches nearly the supersonic speed during intense storms (e.g., Goncharenko et al., 2003). Under such conditions, modeling of the neutral atmosphere based on the assumption of the hydrostatic equilibrium is no longer appropriate. Therefore, we employ a two-dimensional nonhydrostatic compressible atmospheric model to study the behavior of vertical winds associated with a local heat source in a background flow. The modeling method is basically the same as previous nonhydrostatic thermospheric models (Walterscheid et al., 1985; Walterscheid and Lyons, 1992; Sun et al., 1995; Shinagawa et al., 2003).
In order to treat small-scale convective and wave motion accurately, CIP (Cubic-Interpolated Pseudoparticle) method (cf. Takewaki et al., 1985; Yabe et al., 1991; Yabe and Wang, 1991) is adopted for the advection terms in the fluid equations (see Appendix). This numerical scheme is now widely used in various research fields related to fluid dynamics because of its high numerical accuracy in solving advection equations. The domain of the model is 0 (i.e., surface) $-700 \mathrm{~km}$ in altitude ( $z$ axis), and $0-4000 \mathrm{~km}$ in horizontal (meridional) direction ( $y$ axis), where $+y$ direction is taken to be poleward. In longitudinal direction $x$, all physical quantities are assumed to be uniform. The vertical grid size is $4 \mathrm{~km}$, and the horizontal grid size is $5 \mathrm{~km}$. The equations for the neutral atmosphere include the continuity, momentum, energy, and equation of state. These equations are for one mean gas fluid. The composition and specific heats are allowed to vary with altitudes as a prescribed function which is taken from the MSISE-90 model (Hedin, 1991). Input parameters for the neutral atmosphere were taken from MSISE-90 model by setting $69^{\circ} \mathrm{N}, 19^{\circ} \mathrm{E}$, $\mathrm{F} 10.7=88, \mathrm{Ap}=47,5.5$ hours UT, on March 1, 1995. These values are rather arbitrary, and do not significantly affect the results of this study.

The equations are as follows.

Continuity equation:

$$
\frac{\partial \rho}{\partial t}=\frac{\partial(\rho v)}{\partial y}+\frac{\partial(\rho w)}{\partial z}
$$

Momentum equations:

$$
\frac{\partial u}{\partial t}=-v \frac{\partial u}{\partial y}-w \frac{\partial u}{\partial z}+2 \Omega \sin \phi v-v_{n i}\left(u-u_{i}\right)+\frac{1}{\rho} \nabla \cdot(\eta \nabla u)
$$

$$
\begin{aligned}
\frac{\partial v}{\partial t}= & -v \frac{\partial v}{\partial y}-w \frac{\partial v}{\partial z}-2 \Omega \sin \phi u \\
& -\frac{1}{\rho} \frac{\partial p}{\partial y}-v_{n i}\left(v-v_{i}\right)+\frac{1}{\rho} \nabla \cdot(\eta \nabla v)
\end{aligned}
$$

$$
\begin{aligned}
\frac{\partial w}{\partial t}= & -v \frac{\partial w}{\partial y}-w \frac{\partial w}{\partial z}-2 \Omega \cos \phi u \\
& -\frac{1}{\rho} \frac{\partial p}{\partial z}-g-v_{n i}\left(w-w_{i}\right)+\frac{1}{\rho} \nabla \cdot(\eta \nabla w)
\end{aligned}
$$

Energy equation:

$$
\begin{aligned}
\frac{\partial T}{\partial t}= & -v \frac{\partial T}{\partial y}-w \frac{\partial T}{\partial z}-\frac{R}{c_{v}} T \\
& \cdot\left(\frac{\partial v}{\partial y}+\frac{\partial w}{\partial z}\right)+\frac{1}{\rho c_{v}} \frac{\partial}{\partial z}\left(\kappa \frac{\partial T}{\partial z}\right)-\frac{Q}{c_{v}}
\end{aligned}
$$

Equation of state:

$$
p=\rho R T
$$

where $t$ time, $y$ meridional distance, $z$ altitude, $\rho$ mass density, $u$ eastward velocity, $v$ northward velocity, $w$ vertical velocity, $p$ pressure, $R$ gas constant, $T$ temperature, $c_{v}$ specific heat for constant volume, $\eta$ molecular viscosity, $\kappa$ thermal conduction coefficient, $Q$ heating rate, $\phi$ latitude. 


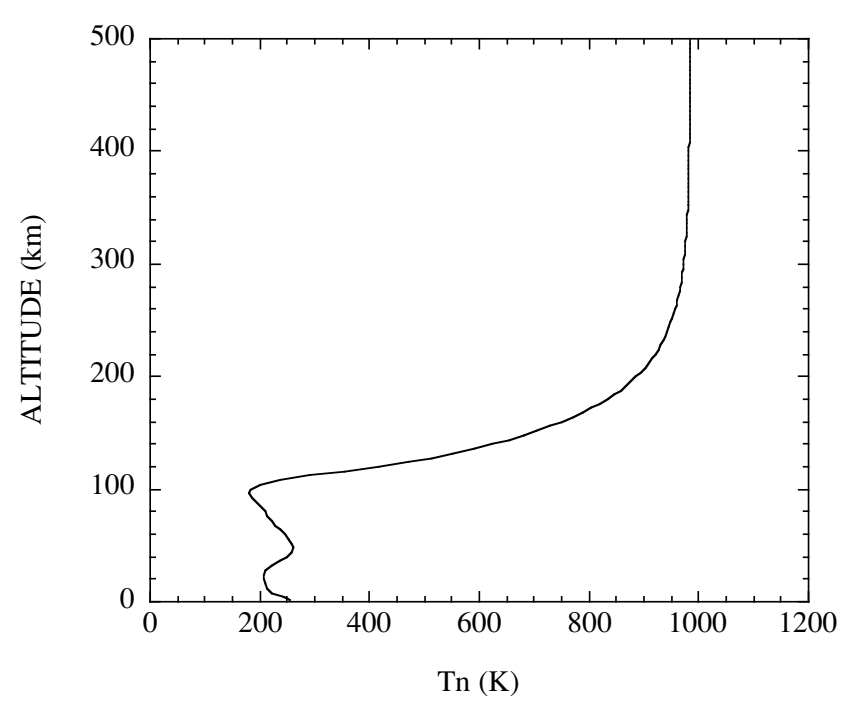

Fig. 1. Vertical profile of initial neutral temperature taken from MSISE-90.

In the actual numerical calculation, perturbation quantities (e.g., $\rho^{\prime}=\rho-\rho_{0}$ ) are solved including nonlinear terms instead of the quantity itself (e.g., $\rho$ ). As mentioned earlier, the background quantities $\left(\rho_{0}, T_{0}, \ldots\right)$ of the neutral atmosphere are taken from the MSISE-90 model. It is assumed in the model that the background atmosphere $\left(\rho_{0}, T_{0}, \ldots\right)$ is in equilibrium for all equations above. The altitude profiles of $T_{0}$, and the neutral densities used in this study are shown in Figs. 1 and 2, respectively. The atmosphere is assumed to be at rest initially $\left(u=u^{\prime}=0, v=v^{\prime}=0, w=w^{\prime}=0\right.$ at $t=0)$. The Coriolis forces, molecular and eddy viscosities, ion drag forces, and thermal conduction are included. The effect of convection electric field on ion motion $(E \times B$ drift), which could accelerate neutral particles through collision, is not included in this model. Composition equations for neutral species are also not included in this calculation. The heating is introduced in the model as an input to the energy equation. The time step used for the calculation is 0.2 seconds for all calculations.

Boundary conditions are given as follows: (1) For mass density $\rho^{\prime}$, free boundary conditions at $z=0$, and $z=$ top, (2) For vertical velocity $w^{\prime}$, mirror boundary condition at $z=0$; no vertical gradient $(\partial w / \partial z=0)$ at $z=$ top, (3) For horizontal velocity $u^{\prime}$, no vertical gradient $(\partial v / \partial z=0)$ at $z=0$, and $z=$ top, (4) For temperature $T^{\prime}$, free at $z=0$; no vertical gradient $(\partial T / \partial z=0)$ at $z=$ top, (5) For all variables, no horizontal gradient $(\partial / \partial y=0)$ at the left boundary $(y=0)$ and the right boundary $(y=4000$ $\mathrm{km})$.

The altitude profile of the background velocity $v_{h}(z)$ is given as follows.

$$
\begin{aligned}
v_{h}(z) & =v_{0} \text { for } z \geq z_{0} ; v_{h}(z) \\
& =v_{0} \exp \left(-\left\{\left(z-z_{0}\right) / h_{v}\right\}^{2}\right) \text { for } z \leq z_{0}
\end{aligned}
$$

where $z$ is altitude, $z_{0}=120 \mathrm{~km}, h_{v}=10 \mathrm{~km}, v_{0}$ is a given constant for each simulation. An additional term $-\left(v-v_{h}(z)\right) / \tau,(\tau=1000 \mathrm{sec})$ is added to the meridional momentum equation (3) as an "external forcing" to drive horizontal background flow. $v_{0}$ is fixed for one simu-

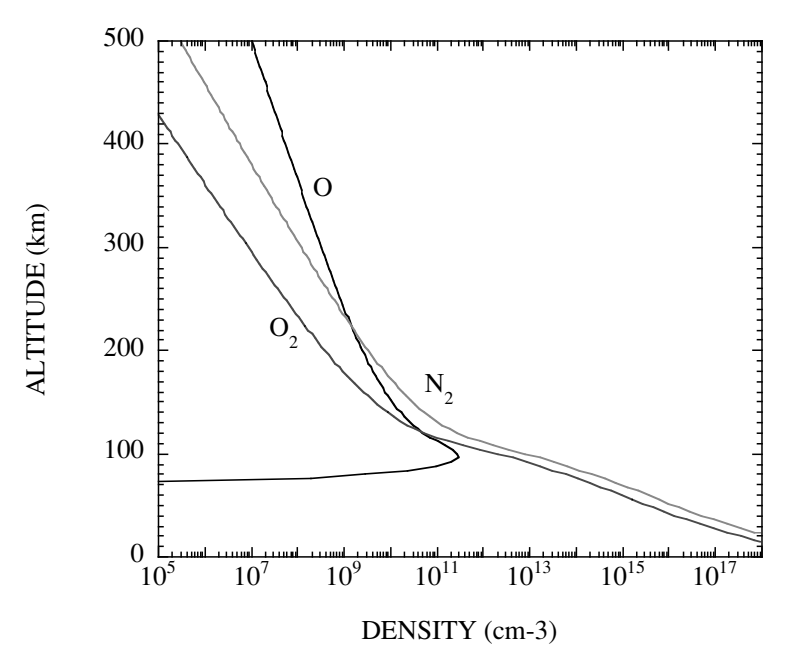

Fig. 2. Vertical profile of initial neutral composition taken from MSISE-90.

HEATING RATE (log-erg/cm3/sec)

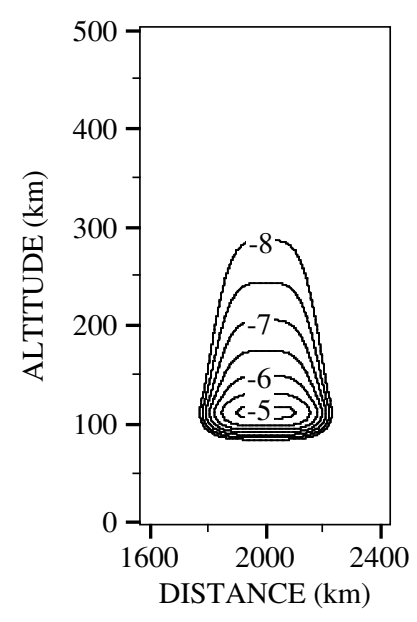

Fig. 3. Contour plot of neutral heating rate used in the calculations.

lation, and several cases between $v_{0}=0 \mathrm{~m} / \mathrm{s}$ and $v_{0}=600$ $\mathrm{m} / \mathrm{s}$ have been studied. We consider it as a general external force, such as pressure gradient or ion drag force, but the meaning of this term is not very much clear. Although more realistic forcing is desirable to obtain more realistic horizontal velocity, the method of this study is good enough for the purpose of this study. In reality, observations of the thermosphere suggest that the horizontal velocity varies significantly with altitude (e.g., Parish et al., 2003). Our test calculations indicate that the dynamics of the neutral atmosphere depends on the altitude profile of this artificial horizontal forcing term, although the overall behavior of the vertical velocity is not significantly affected by the horizontal velocity profile.

Spatial distribution of the heating rate used in the model calculations is shown in a contour plot in Fig. 3. The heating rate profile used in this study is assumed to be Joule heating, and the profile is based on conductivities estimated from the EISCAT data (Brekke et al., 1995; Brekke and Kamide, 1996), and the typical electric field values in the polar region. It is noted that the heating region varies significantly 


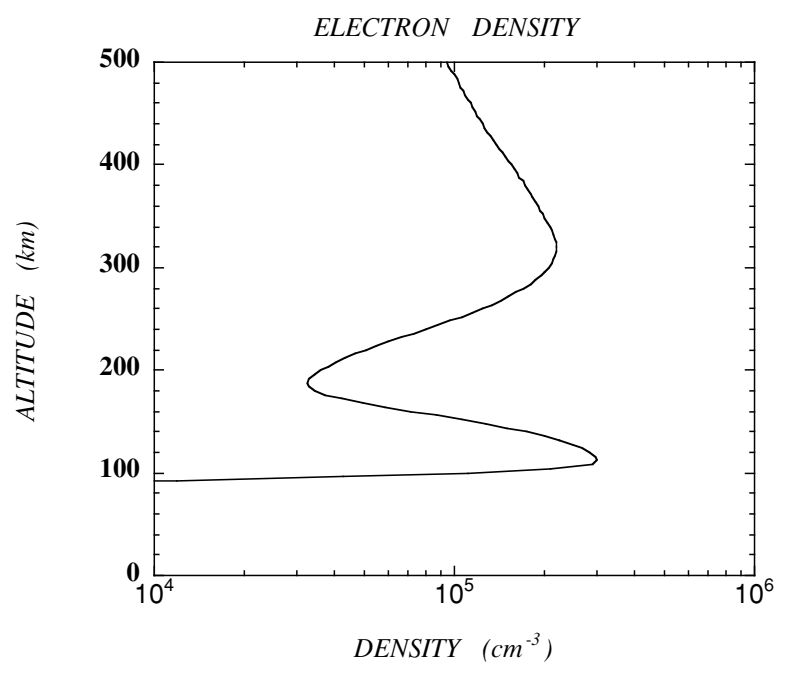

Fig. 4. Altitude profile of electron density used in the model.

(a)

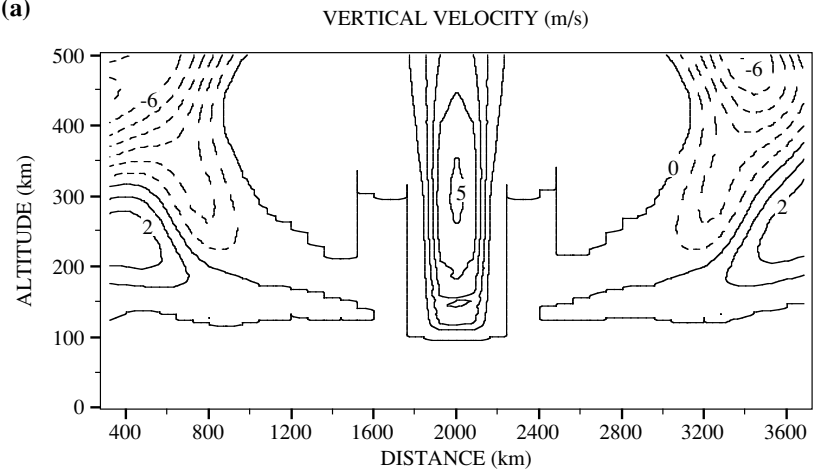

(b)

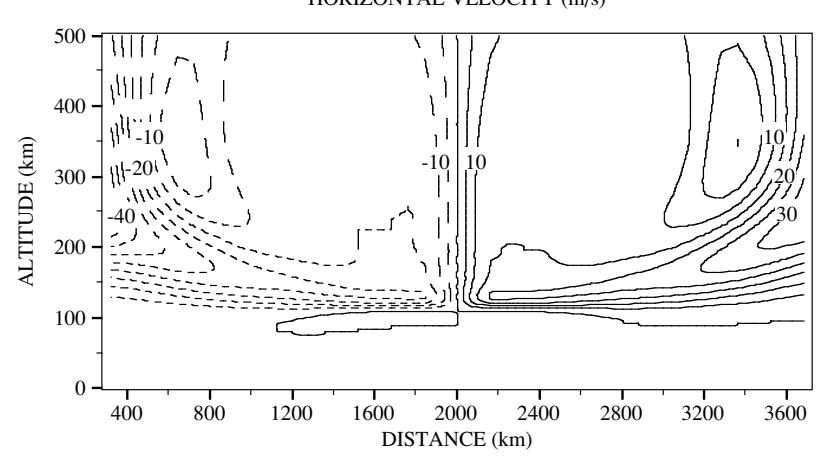

Fig. 5. Contour plots of (a) vertical velocity (plus sign is upward), and (b) horizontal velocity (plus sign is rightward), one hour after heating is introduced without horizontal background flow.

both in time and in space. The heat source is given only in the region between $y=1800 \mathrm{~km}$ and $2200 \mathrm{~km}$, and the center of the heat source is located at $y=2000 \mathrm{~km}$. Since the values in the contour plot are indicated in logarithmic scale, width of the main heating region is practically about $200 \mathrm{~km}$. In the model calculations, the dynamics of the thermosphere associated with this local heating in horizontal background flow is investigated.

The altitude profile of the electron density is shown in Fig. 4. The electron density is assumed uniform in horizontal direction. In reality, there is local enhancement in electron density, which might cause local motion in the neutral (a)

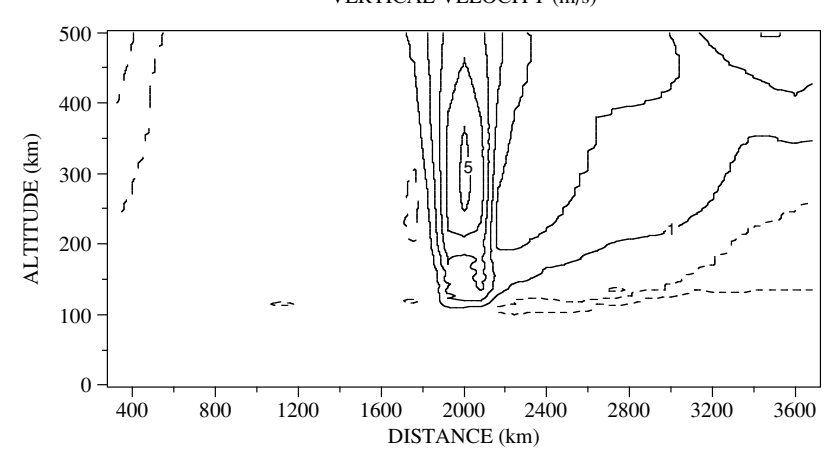

(b)

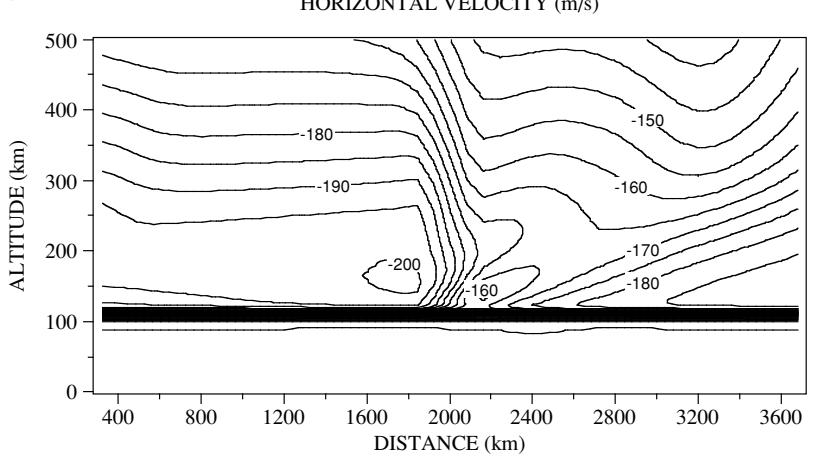

Fig. 6. Contour plots of (a) vertical velocity, and (b) horizontal velocity, one hour after heating is introduced without horizontal background flow introduced with background horizontal flow of about $200 \mathrm{~m} / \mathrm{s}$.

atmosphere through ion-neutral drag. In this study, however, we did not include the effect of local enhancement of ion-neutral drag, which might also play a significant role in the dynamics. The ionosphere is included only as a drag source through ion-neutral collisions. Fixed ionosphere is given (i.e., ions do not move), with collision frequencies based on Schunk and Nagy (1980). However, it was found that when the ion density is less than about $5 \times 10^{5} \mathrm{~cm}^{-3}$, the ion-neutral drag effect on the dynamics of the thermosphere does not play an important role in the present calculations. Thus, it turned out that the ionospheric density profile adopted in this study does not have so much meaning for present simulations.

Starting from the unperturbed atmosphere, in which no horizontal background velocity is given, a heat source and a horizontal background flow are introduced. The heating rate is fixed throughout the calculations. For all cases, after about one hour, velocities in the simulation domain have almost reached steady-state values.

\section{Results}

When the heat source is introduced into the thermosphere, initial perturbation through thermal expansion is driven, and the air in the heated region expands immediately. Consequently, upward motion is driven through thermal expansion. After this initial perturbation, gravity waves are generated and propagate to both horizontal directions. At about an hour, the gravity waves are almost moving out of the simulation domain. Figure 5(a) shows a contour plot of the calculated vertical velocity at one hour after the heat source is introduced. In the heating region, steady upward 
(a)

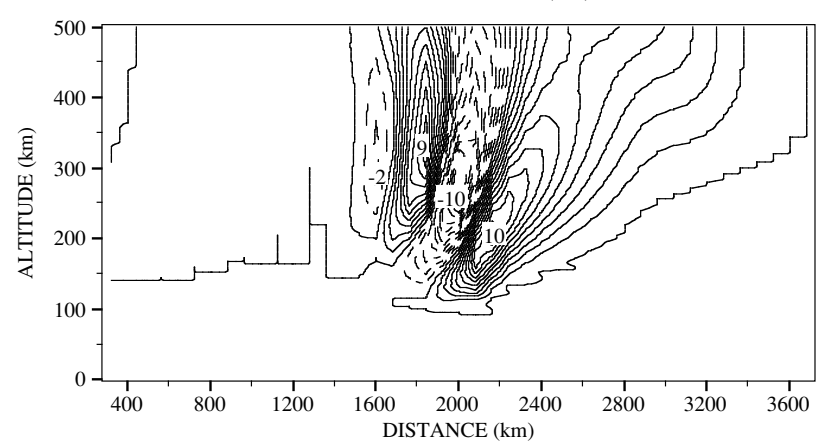

(b)

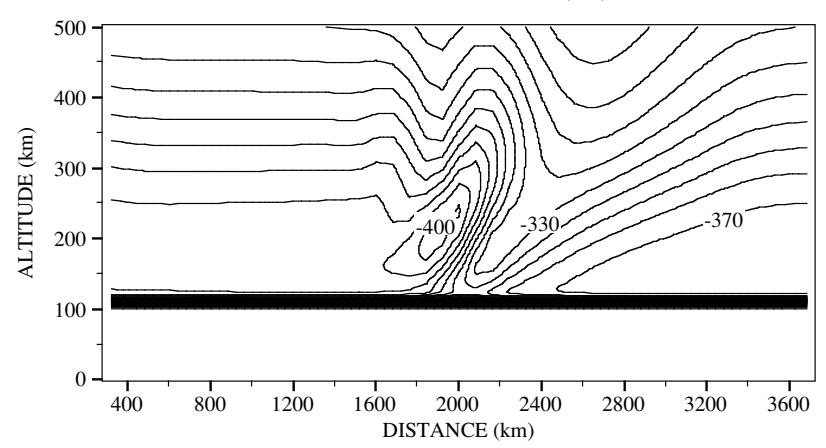

(c)

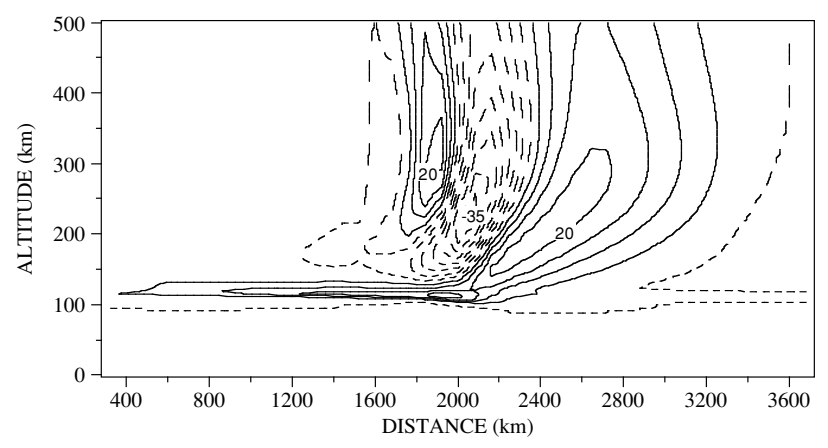

Fig. 7. Contour plots of (a) vertical velocity (plus sign is upward), (b) horizontal velocity (plus sign is rightward), and (c) temperature change, one hour after heating is introduced with background horizontal flow of about $400 \mathrm{~m} / \mathrm{s}$.

motion is generated. The magnitude of the vertical velocity is about $5 \mathrm{~m} / \mathrm{s}$ in the upper thermosphere in the center of the heated region. The horizontal velocity contour is shown in Fig. 5(b). In the horizontal direction, the air expands from the center of the heating region to both directions. The horizontal wind speed in the upper thermosphere near the heated region is about $10 \mathrm{~m} / \mathrm{s}$. In this calculation, no background flow is included.

To study the effect of large-scale horizontal flow on the dynamics of the thermosphere within and near the heated region, an artificial horizontal forcing term is introduced. For all calculations, nearly steady-state values are shown. At first, to introduce background horizontal flow in the thermosphere, $v_{0}$ in Eq. (7) is taken to be $200 \mathrm{~m} / \mathrm{s}$ so that the horizontal flow reaches approximately $200 \mathrm{~m} / \mathrm{s}$ at high altitudes. Figures 6(a) and 6(b) illustrate the calculated vertical and horizontal velocities, respectively, at one hour after the horizontal forcing term is introduced. In Fig. 6(a), posi-

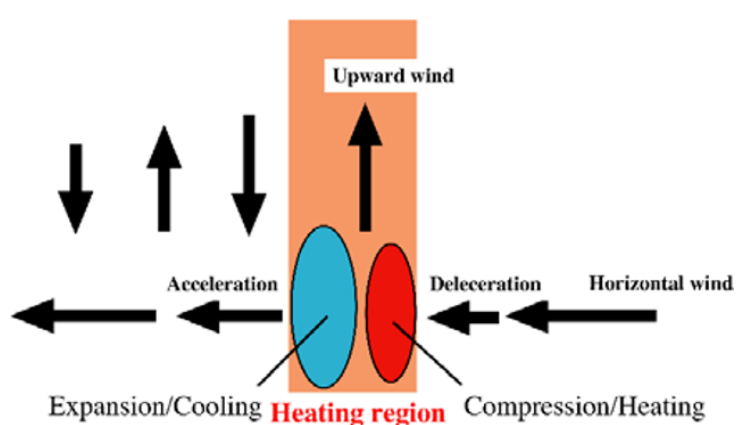

Fig. 8. Schematic picture of interaction between horizontal flow and localized heating region.

tive numbers for contour lines indicate upward velocities. In Fig. 6(b), negative numbers for contour lines indicate leftward velocities. The horizontal velocity is about 150 $200 \mathrm{~m} / \mathrm{s}$ in the upper thermosphere, and the flow direction is from the right-hand side to the left-hand side. The calculated vertical velocity profile is about $5 \mathrm{~m} / \mathrm{s}$ in the heat source, which is almost the same as the vertical velocity in Fig. 5(a). In the upstream region, a weak upward velocity of a few meters per second is formed. A weak downward motion less than $1 \mathrm{~m} / \mathrm{s}$ is generated in the downstream side of the heated region. The horizontal speed decreases, as the air parcel gets closer to the heating region in the upstream. The horizontal velocity at $y=1700 \mathrm{~km}$ is larger than the velocity at $y=2200 \mathrm{~km}$, indicating some acceleration occurred downstream of the heating region with respect to the upstream region. This is caused by the increased pressure near the heated region. Although the horizontal velocity changes significantly, the momentum $\rho v$ is approximately constant horizontally because of the mass density enhancement for low horizontal velocity regions.

In the next case, an artificial horizontal flow speed $v_{0}$ is set to be $400 \mathrm{~m} / \mathrm{s}$. Figures 7(a) and 7(b) illustrate the calculated vertical and horizontal velocities, respectively, at one hour after the forcing term is introduced. The horizontal velocity becomes about $350-400 \mathrm{~m} / \mathrm{s}$ in the upper thermosphere. It is found that the vertical velocity profile is altered significantly compared with the vertical velocity in the previous case (Fig. 5(a)). The maximum upward motion reaches $10 \mathrm{~m} / \mathrm{s}$, and a downward motion of $-10 \mathrm{~m} / \mathrm{s}$ is formed in the downstream side of the heating region. Then the velocity becomes upward again. An oscillation of the vertical velocity appears in the downward region of the heat source. The horizontal flow decelerates in the upstream region of the heating region, and goes back to the initial velocity in the downstream side (Fig. 7(b)). At high altitudes, the vertical flow is upward in the upstream region as well as in the heating region. A weak downward flow region appears in the downstream region.

Although this is a snapshot at one hour after introducing the horizontal flow, this structure becomes almost steadystate. The speed of the horizontal background flow is now close to the sonic speed in the lower thermosphere, while the horizontal flow at high altitudes is still smaller than the sonic speed. There is a significant interaction between the background horizontal flow and the local upward flow. The 
(a)

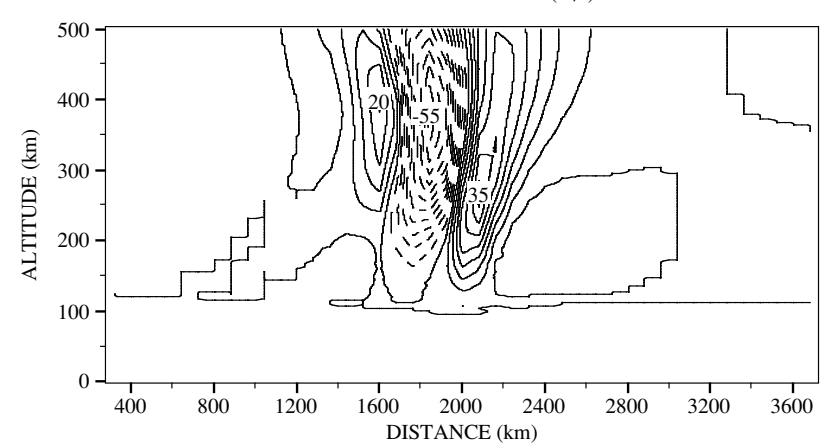

(b)

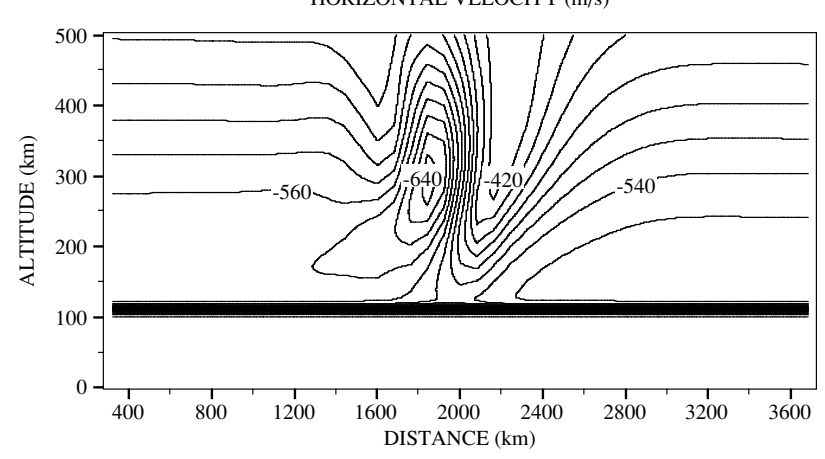

Fig. 9. Contour plots of (a) vertical velocity, and (b) horizontal velocity, one hour after heating is introduced with background horizontal flow of about $600 \mathrm{~m} / \mathrm{s}$.

temperature perturbation is shown in Fig. 7(c).

Figure 8 schematically illustrates the dynamics and energetics in the vicinity of the heated region in the horizontal flow. In the upstream region of the heat source, temperature increases because of compression of the horizontal flow (red region). The air in the heating region tends to expand from the heated region, which collides with the background motion. The temperature decreases within and downstream region of the heated region (blue region) partly because the upward motion moves the cooler air of the lower thermosphere, and partly because the atmosphere expands in the downstream region.

In the last case, the horizontal background flow is raised up to about $600 \mathrm{~m} / \mathrm{s}$ in the upper thermosphere. Vertical and horizontal velocities at one hour are shown in Figs. 9(a) and 9(b), respectively. Upward flow in the heating region is now more than $30 \mathrm{~m} / \mathrm{s}$, which is larger than the previous case. A downward flow appears in the region between $y=1600$ $\mathrm{km}$ and $1900 \mathrm{~km}$. The maximum downward velocity is about $-55 \mathrm{~m} / \mathrm{s}$. At above $250 \mathrm{~km}$, another upward region appears in the downstream side of the downward region. Strong wave-like structure of the vertical wind appears. As mentioned later, for this case the horizontal wind speed exceeds the sonic speed in the lower thermosphere, and acoustic waves are expected to be generated in addition to gravity waves.

\section{Discussion}

Previous observational and theoretical studies have suggested that upward vertical winds in the thermosphere may be generated by Joule or particle heating in the auroral re-

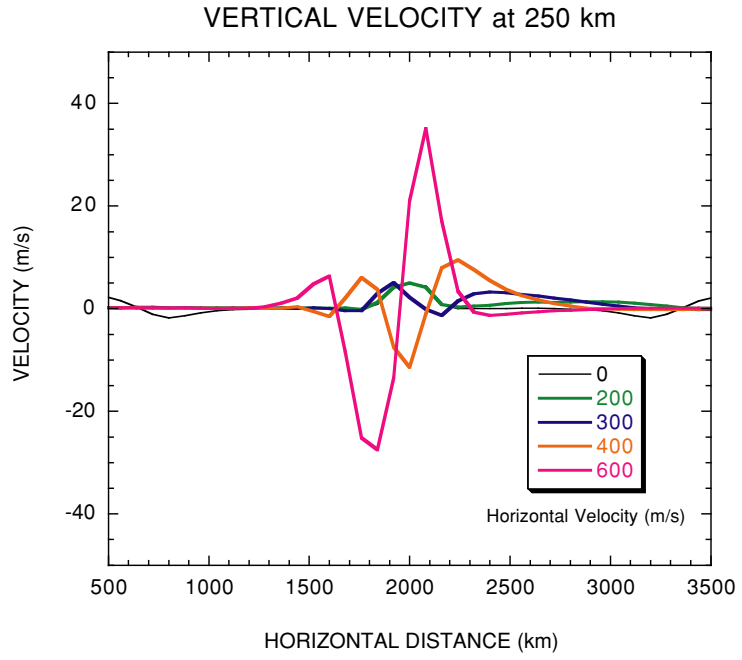

Fig. 10. Vertical velocities at an altitude of $250 \mathrm{~km}$ at one hour after the start of heating for background horizontal winds of 0, 200, 300, 400, and $600 \mathrm{~m} / \mathrm{s}$.

gion. But, the region of the upward wind does not always coincide with the most heated region, indicating that the interaction between the local heating and horizontal background plays an important role in the dynamics in the vicinity of an auroral arc. Some observations imply that the upward motion tends to appear in the poleward side of the auroral oval, and that downward motion appears in the equatorward of the auroral oval (Ishii et al., 2001; Ishii, 2005; Oyama et al., 2003, 2005). Smith (2000) suggested that waves of vertical winds can be generated in the downstream region associated with auroral heating. Shinagawa et al. (2003) studied the dynamics near a moving auroral arc, using a two-dimensional thermospheric model. They found that wave-like signature tends to be formed in the downstream region of the moving heat source. However, their study showed only a few snapshots of the velocity near an arc, which might not be a typical or steady feature.

In this study, the interaction between a local heat source and a background horizontal flow was investigated in more detail than Shinagawa et al. (2003). It is found that for large horizontal flow larger than $300 \mathrm{~m} / \mathrm{s}$, this picture agrees qualitatively to the model calculations obtained in this study. For the small horizontal flow less than $300 \mathrm{~m} / \mathrm{s}$, this effect is not significant. When the horizontal flow is large $\left(v_{0}>600\right.$ $\mathrm{m} / \mathrm{s})$, significant wave-like motion of the vertical wind is formed. The calculated vertical velocity at the altitude of $250 \mathrm{~km}$ for $v_{0}=0,200,300,400$, and $600 \mathrm{~m} / \mathrm{s}$ are shown in Fig. 10. This result indicates that the magnitude of the vertical velocity near the heat source is not linearly proportional to the background horizontal wind speed.

The dynamics and energetics of gravity waves generated by the neutral flow passing the heating region are extremely complicated especially when the flow speed is near the sonic speed. Acoustic-gravity waves radiated from moving sources were investigated by Kato et al. (1977) and Kato (1980). They employed a linear analysis to study gravity wave emission from a moving line source as well as a moving point source in the upper atmosphere. Subsonic, transonic, and supersonic cases for the moving sources were 


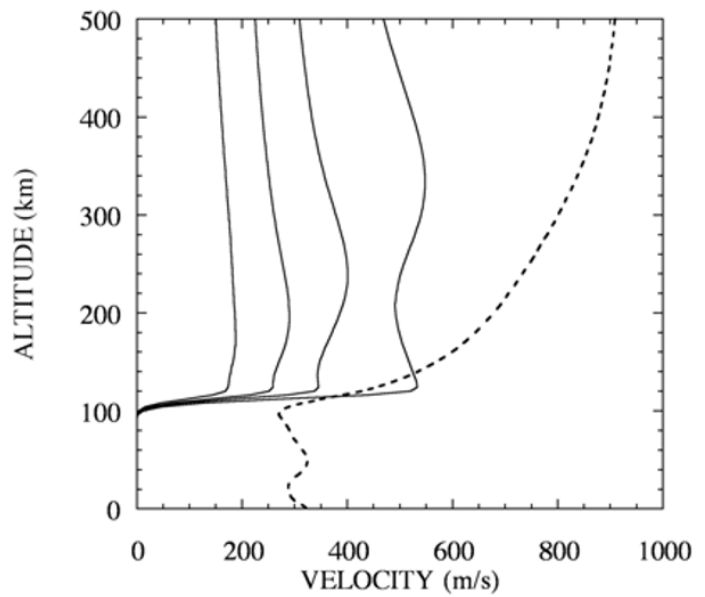

Fig. 11. Altitude profile of the horizontal flow speeds (solid lines) at the center of the heat source $(y=2000 \mathrm{~km})$ for four cases $(v \sim 200$ $\mathrm{m} / \mathrm{s}, 300 \mathrm{~m} / \mathrm{s}, 400 \mathrm{~m} / \mathrm{s}$, and $600 \mathrm{~m} / \mathrm{s}$ ), and the sonic speed (broken line). For $600 \mathrm{~m} / \mathrm{s}$ case, the flow speed exceeds the sonic speed in the region between 110 and $120 \mathrm{~km}$, where waves with acoustic mode are generated.

studied.

It is noted that the studies of Kato et al. (1977) and Kato (1980) are not able to give actual velocity fields because (1) a point source or a line source is assumed, (2) nature of the source is not specified, (3) linearity of the system, i.e., small amplitude is assumed, and (4) the realistic atmosphere is not included. Nonetheless, they successfully showed that when the speed of the source becomes closer to the sonic speed, the physical quantities jump suddenly as the source passes the observation point, indicating strong compression occurs in front of the moving source. When the source moves larger than the sonic speed, i.e., the flow is supersonic, even larger jump is generated, followed by the high-frequency oscillation. The frequency for subsonic case, i.e., slow flow case, is close to $\omega_{g}$, while the period of the oscillation for supersonic case is larger than $\omega_{a}$. That is, the period of the oscillation is shorter than the Brunt-Väisälä period. When Mach number is close to one, i.e., near the singular point, it is hard to understand theoretically how the wave behaves. We plotted the horizontal flow speeds and sonic speed in Fig. 11. It is seen that for the horizontal velocity of $400 \mathrm{~m} / \mathrm{s}$, there is a region where the flow speed becomes very close to the sonic speed. For velocity of $600 \mathrm{~m} / \mathrm{s}$, this is a region where the flow speed exceeds the sonic speed. This result indicates that the oscillation is expected for $v=400 \mathrm{~m} / \mathrm{s}$ case, and strong oscillation is expected for $600 \mathrm{~m} / \mathrm{s}$ case.

Figure 12 shows the dispersion diagram for acousticgravity waves. A linear analysis by Kato et al. (1977) and by Kato (1980) shows that when the source moves at the supersonic speed (line a), acoustic-mode waves are radiated from the source. When the source speed is exactly the same as the sonic speed (line b), the Lamb wave is radiated. When the source speed is subsonic (line c), gravity waves are generated. In the calculation of our study, however, the flow speed and the sonic speed are altitude-dependent. Thus, different region emits a different type of acousticgravity wave. The resultant wave is composition of all

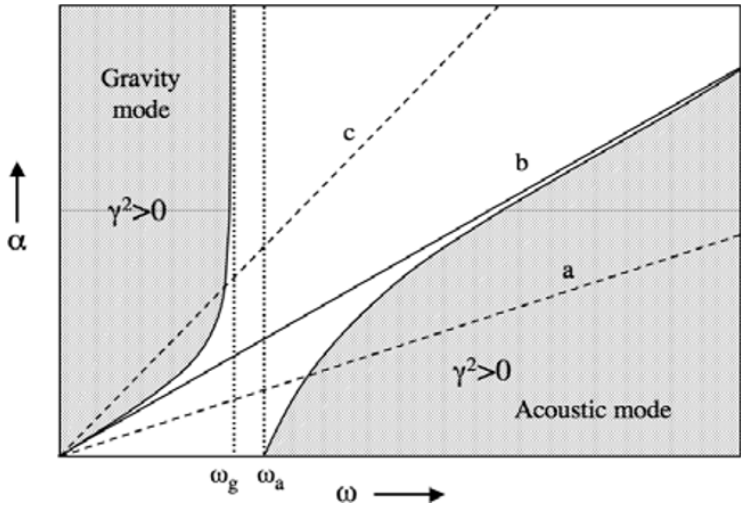

Fig. 12. Dispersion diagram for acoustic-gravity waves, where $\alpha$ and $\gamma$ are waves numbers, in the $y$ (horizontal) and $z$ (vertical) directions, respectively. $\omega_{g}=$ Brunt-Väisälä frequency, $\omega_{a}=$ acoustic cutoff frequency. $\alpha$ versus $\omega$ is shown with $\gamma$ as parameter. The internal waves as $\gamma^{2}>0$ exist only in the shaded region. The slant lines a, b, and $\mathrm{c}$ are given by $=V_{0} \alpha$, where the horizontal flow speed $V_{0}>C s$ (supersonic flow), $V_{0}=C s$ (sonic), and $V_{0}<C s$ (subsonic), respectively.

possible waves generated at every point, and it is difficult to specify what kinds of waves finally remain at a later time. In particular, the flow velocity for $v=600 \mathrm{~m} / \mathrm{s}$ case exceeds the sonic speed at some altitudes where acoustic waves (bow waves) are emitted instead of gravity waves, giving a stronger oscillation near the heating region (Kato et al., 1977; Kato, 1980). Strong and sudden pressure jump occurs in front of the heating region, and sudden decrease behind the heating region, which results in a large upward and downward motion near the arc.

In our study, the heat source is not a line source, but has a finite width in $y$-direction. The horizontal size of the heat source may also affect the behavior of the vertical motion. The moving line source suggests an oscillation with the Brunt-Väisälä frequency for subsonic flow case, and a higher frequency for supersonic flow case. The line source approximation is valid if the horizontal scale of the heat source is much smaller than $V_{0} / \omega_{g}$, where $V_{0}$ is a flow speed. In the present simulation, for $v_{0}<300 \mathrm{~m} / \mathrm{s}$ case, the line-source approximation is not very good, which might be one of the reasons for small vertical oscillation.

Furthermore, there are other important factors, which control the dynamics of the auroral thermosphere. First, three-dimensional effects need to be included because a heat source usually has a structure in both horizontal directions. Second, strong electric fields exist in the polar ionosphere, which drives large ion motion and accelerates the thermosphere. Third, altitude profile of the heat source is still not fully understood, especially in the lower thermosphere. Finally, more realistic altitude profile of the horizontal flow needs to be included. In the lower thermosphere, a strong shear of the neutral wind is occasionally observed, which may significantly modify the vertical wind profile.

\section{Conclusions}

The behavior of the vertical motion near an auroral heating region embedded in the background horizontal flow has been studied in order to examine whether or not wave-like 
motion in the downward region proposed by Smith (2000) and by Shinagawa et al. (2003). It was found that significant vertical motion could be generated by a combination of local heating and the horizontal flow if the flow speed is larger than about $300 \mathrm{~m} / \mathrm{s}$. Our simulation study suggests that structure of vertical wind becomes oscillatory when both heating region and large horizontal flow exist.

The observed vertical wind sometimes exhibits rather persisting upward or downward motion even when the heating is relatively weak or when horizontal velocity is not very large. Although some feature of the vertical motion is qualitatively reproduced in this study, it is necessary to include various effects such as ion-neutral drag driven by large electric field in the ionosphere, temporal and spatial variations in heat source, three-dimensional patterns of neutral wind system in order to understand quantitatively the observed behavior of the vertical motion.

As we described in Discussion, the most important parameters to understand effects of the auroral heating on the polar thermosphere are time-dependent three-dimensional data of the horizontal neutral wind as well as the heating rate. The most effective way to derive those parameters from observations is to use data obtained by in situ rocket measurements, the IS radar, the VHF radar, all-sky FPIs, all-sky imagers, photometers, etc. In our future studies, we plan to examine our results by comparing our theoretical results with data obtained by the DELTA (Dynamics and Energetics in the Lower Thermosphere in Aurora) campaign (cf., Abe et al., 2006). We also plan to use a threedimensional model of the thermosphere to reproduce the small-scale dynamics near an auroral arc.

Acknowledgments. This work was carried out by the joint research program of the Solar-Terrestrial Environment Laboratory, Nagoya University. This work was supported by a Grant-in-Aid from the Ministry of Education, Culture, Sports, Science and Technology of Japan (No. 16654075).

\section{Appendix.}

We present a brief summary of the CIP (cubicinterpolated pseudoparticle) scheme (for more detail, refer to Takewaki et al. (1985), Yabe et al. (1991), and Yabe and Wang (1991)). The following hyperbolic equation is considered:

$$
\frac{\partial f}{\partial t}+u \frac{\partial f}{\partial x}=G
$$

where $u$ is the advection velocity, and the source term G can be a function of $f$. We separate the above equation into two parts: the nonadvection part, $\partial f / \partial t=G$, and the advection part, $\partial f / \partial t+u \partial f / \partial x=0$.

Taking the spatial derivative of Eq. (A.1),

$$
\frac{\partial f^{\prime}}{\partial t}+u \frac{\partial f^{\prime}}{\partial x}=G^{\prime}-f^{\prime} \frac{\partial u}{\partial x}
$$

where a prime denotes the spatial derivative about $x$. This equation can also be separated into two parts: $\partial f / \partial t=G^{\prime}-$ $f^{\prime} \partial u / \partial x$ (non-advection part) and $\partial f^{\prime} / \partial t+u \partial f^{\prime} / \partial x=0$ (advection part). The CIP scheme is applied to the advection parts of Eqs. (A.1) and (A.2) after the non-advection parts are solved with a finite difference approach.

\section{A.1 Non-advection part}

The equation for $f$, i.e., $\partial f / \partial t=G$, is simply solved by using finite differencing,

$$
f_{i}^{*}=f_{i}^{n}+G_{i} \Delta t
$$

where the asterisk on $f$ indicates the time after one time step in the nonadvection part. The time evolution of $f^{\prime}$ associated with $G^{\prime}$ can be estimated from

$$
\begin{aligned}
\frac{f_{i}^{\prime *}-f_{i}^{\prime n}}{\Delta t}= & \frac{f_{i+1}^{\prime *}-f_{i-1}^{\prime *}-f_{i+1}^{\prime n}+f_{i-1}^{\prime n}}{2 \Delta x \Delta t} \\
& -f_{i}^{\prime n} \frac{u_{i+1}-u_{i-1}}{2 \Delta x}
\end{aligned}
$$

where the spatial derivative of $G$ is approximated as (using Eq. (A.3))

$$
\frac{G_{i+1}-G_{i-1}}{2 \Delta t}=\frac{f_{i+1}^{*}-f_{i-1}^{*}-f_{i+1}^{n}+f_{i-1}^{n}}{2 \Delta x \Delta t}
$$

This approximation is useful because Eq. (A.4) does not contain $G$, which generally includes higher order spatial derivatives and nonlinear terms.

\section{A.2 Advection part}

After $f$ and $f^{\prime}$ are advanced in the non-advection part, the CIP scheme is applied to the advection part,

$$
\frac{\partial f^{*}}{\partial t}+u \frac{\partial f^{*}}{\partial x}=0
$$

Even if $u$ is a function of $t$ and $x$, we can locally use the solution

$$
f^{*}\left(x_{i}, t\right) \sim f *\left(x_{i}-u * \Delta t, t-\Delta t\right)
$$

Since the value of $f^{*}$ is given only at grid points, $f^{*}\left(x_{i}-\right.$ $\left.u^{*} \Delta t, t-\Delta t\right)$ is approximated by the cubic polynomial interpolation

$$
F_{i}(x)=\left[\left(a_{i} X+b_{i}\right) X+f_{i}^{\prime *}\right] X+f_{i}^{*}
$$

where $X=x-x_{i}$. We require that the value $f^{*}$ and the spatial derivative $f^{\prime *}$ should be continuous at the grid points $i+1$. As a result, we have

$$
\begin{aligned}
& a_{i}=\frac{f_{i}^{\prime *}+f_{i+1}^{\prime *}}{\Delta x^{2}}+\frac{2\left(f_{i}^{*}-f_{i+1}^{*}\right)}{\Delta x^{3}}, \\
& b_{i}=\frac{3\left(f_{i+1}^{*}-f_{i}^{*}\right)}{\Delta x^{2}}-\frac{2\left(f_{i}^{\prime *}+f_{i+1}^{\prime *}\right)}{\Delta x}
\end{aligned}
$$

Once $a_{i}$ and $b_{i}$ are given in terms of $f^{* n}$ and $f^{\prime * n}$, the values of $f^{n+1}$ and $f^{\prime n+1}$ are simply obtained by shifting the cubic polynomial as in Eq. (A.7). Thus

$$
f_{i}^{n+1}=F_{i}\left(x_{i}-u \Delta t\right)=\left[\left(a_{i} \xi+b_{i}\right) \xi+{f_{i}^{\prime *}}^{*}\right] \xi+f_{i}^{*}
$$

$$
{f^{\prime}}_{i}^{n+1}=d F_{i}\left(x_{i}-u \Delta t\right) / d x=\left(3 a_{i} \xi+2 b_{i}\right) \xi+{f^{\prime}}_{i}^{*}
$$

where $\zeta=-u \Delta t$. This expression is derived for $u<0$. For $u>0$, we obtain a similar expression by simply replacing $\Delta x$ by $-\Delta x$ and $i+1$ by $i-1$.

These two parts complete one time step of the calculation; then, after replacing $f^{n+1}$ and $f^{\prime n+1}$ by $f^{n}$ and $f^{\prime n}$, we return to the non-advection part again. 


\section{References}

Abe, T., J. Kurihara, N. Iwagami, S. Nozawa, Y. Ogawa, R. Fujii, H. Hayakawa, and K. Oyama, Dynamics and Energetics in the Lower Thermosphere in Aurora (DELTA)_Japanese sounding rocket campaign-, Earth Planets Space, 58, this issue, 1165-1171, 2006.

Brekke, A. and Y. Kamide, On the relationship between Joule and frictional heating in the polar ionosphere, J. Atmos. Terr. Phys., 58, 139144, 1996.

Brekke, A., S. Nozawa, and M. Sato, Samples of auroral E-region parameters derived from EISCAT experiments, J. Geomag. Geoelectr., 47, 889909, 1995.

Burnside, R. G., F. A. Herrero, J. W. Jr. Meriwether, and J. C. G. Walker, Optical observations of thermospheric dynamics at Arecibo, J. Geophys. Res., 86, 5532-5540, 1981.

Conde, M. and P. L. Dyson, Thermospheric vertical winds above Mauson, Antarctica, J. Atmos. Terr. Phys., 57, 589-596, 1995.

Conde, M., J. D. Craven, T. Immel, E. Hoch, H. Stenbaek-Nielsen, T. Hallinan, R. W. Smith, J. Olson, and W. Sun, Assimilated observations of thermospheric winds, the aurora, and ionospheric currents over Alaska, J. Geophys. Res., 106, 10,493-10,508, 2001.

Eastes, R. W., T. L. Killeen, Q. Wu, J. D. Winningham, W. R. Hoegy, L. E. Wharton, and G. R. Carignan, An experimental investigation of thermospheric structure near an auroral arc, J. Geophys. Res., 97, 10,53910,549, 1992.

Fujiwara, H., S. Maeda, H. Fukunishi, T. J. Fuller-Rowell, and D. S. Evans, Global variations of thermospheric winds and temperatures caused by substorm energy injection, J. Geophys. Res., 101, 225-239, 1996.

Fuller-Rowell, T. J., A Two-dimensional, high-resolution, nested-grid model of the thermosphere 1. Neutral response to an electric field "Spike", J. Geophys. Res., 89, 2971-2990, 1984.

Fuller-Rowell, T. J., A two-dimensional, high-resolution, nested-grid model of the thermosphere 2. Response of the thermosphere to narrow and broad electrodynamic features, J. Geophys. Res., 90, 6567-6586, 1985.

Goncharenko, L. P., J. E. Salah, J. C. Foster, and C. Huang, Variations in lower thermosphere dynamics at midlatitudes during intense geomagnetic storms, J. Geophys. Res., 109, A04304, doi:10.1029/ 2003JA010244, 2003.

Hajkowicz, L. A. and R. D. Hunsucker, A simultaneous observation of large-scale periodic TIDs in both hemispheres following an onset of auroral disturbances, Planet. Space Sci., 35, 785-791, 1987.

Hedin, A. E., Extension of the MSIS thermosphere model into the middle and lower atmosphere, J. Geophys. Res., 96, 1159-1172, 1991.

Innis, J. L. and M. Conde, Characterization of acoustic-gravity waves in the upper thermosphere using Dynamics Explorer 2 Wind and Temperature Spectrometer (WATS) and Neutral Atmosphere Composition Spectrometer (NACS) data, J. Geophys. Res., 107, A12, 1418, doi:10.1029/2002JA009370, 2002.

Ishii, M., Relationship between thermospheric vertical wind and the location of ionospheric current in the polar region, Adv. Polar Upper Atmos. Res., 19, 63-70, 2005.

Ishii, M., M. Conde, R. W. Smith, M. Krynicki, E. Sagawa, and S. Watari, Vertical wind observations with two Fabry-Perot interferometers at Poker Flat, Alaska, J. Geophys. Res., 106, 10,537-10,551, 2001.

Johnson, F. S., W. B. Hanson, R. R. Hodges, W. R. Coley, G. R. Cargnan, and N. W. Spencer, Gravity waves near $300 \mathrm{~km}$ over the polar caps, $J$. Geophys. Res., 100, 23,993-24,002, 1995.

Kato, S., Chapter 2: Acoustic gravity waves (AGW's), in Dynamics of the Upper Atmosphere, by T. Rikitake, D. Reidel Publishing Company, 1980.

Kato, S., T. Kawakami, and D. St. John, Theory of gravity wave emission from moving sources in the upper atmosphere, J. Atmos. Terr. Phys., 39, 581-588, 1977.

Killeen, T. L., R. A. Heelis, P. B. Hays, N. W. Spencer, and W. B. Hanson, Neutral motions in the polar thermosphere for northward interplanetary magnetic field, Geophys. Res. Lett., 12, 159-162, 1985.

Lyons, L. R. and R. L. Walterscheid, Generation of auroral omega bands by shear instability of the neutral winds, J. Geophys. Res., 90, 12,32112,329, 1985.

Maeda, S., T. J. Fuller-Rowell, and D. S. Evans, Zonally averaged dynamical and compositional response of the thermosphere to auroral activity during September 18-24, 1984, J. Geophys. Res., 94, 16,869-16,883, 1989.

McCormac, F. G., T. L. Killeen, E. Gombosi, P. B. Hays, and N. W. Spencer, Configuration of the high-latitude thermosphere neutral circu- lation for IMF By negative and positive, Geophys. Res. Lett., 12, 155$158,1985$.

Oyama, S., S. Nozawa, S. Maeda, Y. Murayama, R. Fujii, and H. Shinagawa, Field-aligned ion motions in the polar E-F transition region: Mean characteristics, J. Geophys. Res., 108, doi:10.1029/2003JA009830, 2003.

Oyama, S., B. J. Watkins, S. Nozawa, S. Maeda, and M. Conde, Vertical ion motions observed with incoherent-scatter radars in the polar lower ionosphere, J. Geophys. Res., 110, doi:10.1029/2004JA010705, 2005.

Parish, H. F., R. L. Walterscheid, P. W. Jones, and L. R. Lyons, Simulations of the thermospheric response to the diffuse aurora using a threedimensional high-resolution model, J. Geophys. Res., 108, A4, 1140, doi:10.1029/2002JA009610, 2003.

Price, G. D., R. W. Smith, and G. Hernandez, Simultaneous measurements of large vertical winds in the upper and lower thermosphere, J. Atmos. Terr. Phys., 57, 631-643, 1995.

Rees, D., R. W. Smith, P. J. Charleton, F. G. McCormac, N. Lloyd, and A. Steen, The generation of vertical thermospheric winds and gravity waves at auroral latitudes I. Observations of vertical winds, Planet. Space Sci., 32, 667-684, 1984.

Rees, D., T. J. Fuller-Rowell, R. Gordon, M. F. Smith, N. C. Maynard, J. P. Heppner, N. W. Spencer, and L. Wharton, A theoretical and empirical study of the response of the high latitude thermosphere to the sense of the ' $\mathrm{Y}$ ' component of the interplanetary magnetic field, Planet. Space Sci., 34, 1-40, 1986.

Richmond, A. D. and S. Matsushita, Thermospheric response to a magnetic substorm, J. Geophys. Res., 80, 2839-2850, 1975.

Schunk, R. W. and A. F. Nagy, Ionosphere of the terrestrial planets, Rev. Geophys. Space Phys., 18, 813-852, 1980.

Shinagawa, H., S. Oyama, S. Nozawa, S. C. Buchert, R. Fujii, and M. Ishii, Thermospheric and ionospheric dynamics in the auroral region, Adv. Space Res., 31(4), 951-956, 2003.

Sica, R. J., M .H. Rees, J. Romick, G. Hernandez, and R. G. Roble, Auroral zone thermospheric dynamics 1. Averages, J. Geophys. Res., 91, 32313244, 1986.

Smith, R. W., The global-scale effect of small-scale thermospheric disturbances, J. Atmos. Solar-Terr. Phys., 62, 1623-1628, 2000.

Smith, R. W. and G. Hernandez, Vertical winds in the thermosphere within the polar cap, J. Atmos. Terr. Phys., 57, 611-620, 1995.

Sojka, J. J., R. W. Schunk, M. David, J. L. Innis, P. A. Greet, and P. L. Dyson, A theoretical model study of F-region response to high latitude neutral wind upwelling events, J. Atmos. Sol.-Terr. Phys., 63, 15711584, 2001.

Spencer, N. W., L. E. Wharton, H. B. Niemann, A. E. Hedin, G. R. Carignan, and J. C. Maurer, The Dynamics Explorer wind and temperature spectrometer, Space Sci. Inst., 5, 417-428, 1981.

Sun, Z. P., R. P. Turco, R. L. Walterscheid, S. V. Venkateswaran, and P. W. Jones, Thermospheric response to morningside diffuse aurora: High-resolution three-dimensional simulations, J. Geophys. Res., 100, 23,779-23,793, 1995.

Takewaki, H., A. Nishiguchi, and T. Yabe, Cubic Interpolated Pseudoparticle Method (CIP) for solving hyperbolic type equations, J. Comput. Phys., 61, 261-268, 1985.

Thayer, J. P., T. L. Killeen, F. G. McCormac, C. R. Tschan, J.-J. Ponthieu, and N. W. Spencer, Thermospheric neutral wind signatures dependent on the east-west component of the interplanetary magnetic field for northern and southern hemispheres as measured from Dynamics Explorer-2, Ann. Geophys., 5A, 363-368, 1987.

Walterscheid, R. L. and L. R. Lyons, The neutral E region zonal winds during intense postmidnight diffuse aurora: Response to observed particle fluxes, J. Geophys. Res., 94, 3703-3712, 1989.

Walterscheid, R. L. and L. R. Lyons, The neutral circulation in the vicinity of a stable auroral arc, J. Geophys. Res., 97, 19,489-19,499, 1992.

Walterscheid, R. L., L. R. Lyons, and K. E. Taylor, The perturbed neutral circulation in the vicinity of a symmetric stable auroral arc, J. Geophys. Res., 90, 12,235-12,248, 1985.

Yabe, T. and P. Y. Wang, Unified numerical procedure for compressible and incompressible fluid, J. Phys. Soc. Japan, 60, 2105-2108, 1991.

Yabe, T., T. Ishikawa, P. Y. Wang, T. Aoki, Y. Kadota, and F. Ikeda, A universal solver for hyperbolic equations by cubic-polynomial interpolation II. Two- and three-dimensional solvers, Comput. Phys. Commun., 66, 233-242, 1991.

H. Shinagawa (e-mail: sinagawa@nict.go.jp) and S. Oyama 